Bond University

Research Repository

\title{
Awareness, perceptions and practices regarding cancer-related malnutrition and sarcopenia: a survey of cancer clinicians
}

Kiss, Nicole; Bauer, Judy; Boltong, Anna; Brown, Teresa; Isenring, Liz; Loeliger, Jenelle; Steer, Belinda; Findlay, Merran

Published in:

Supportive Care in Cancer

DOI:

10.1007/s00520-020-05371-7

Licence:

Other

Link to output in Bond University research repository.

Recommended citation(APA):

Kiss, N., Bauer, J., Boltong, A., Brown, T., Isenring, L., Loeliger, J., Steer, B., \& Findlay, M. (2020). Awareness, perceptions and practices regarding cancer-related malnutrition and sarcopenia: a survey of cancer clinicians. Supportive Care in Cancer, 28(11), 5263-5270. https://doi.org/10.1007/s00520-020-05371-7

\section{General rights}

Copyright and moral rights for the publications made accessible in the public portal are retained by the authors and/or other copyright owners and it is a condition of accessing publications that users recognise and abide by the legal requirements associated with these rights.

For more information, or if you believe that this document breaches copyright, please contact the Bond University research repository coordinator 


\title{
Awareness, perceptions and practices regarding cancer-related malnutrition and sarcopenia: a survey of cancer clinicians
}

\author{
Nicole Kiss ${ }^{1,2}$, Judy Bauer ${ }^{3}$, Anna Boltong ${ }^{4,5}$, Teresa Brown ${ }^{3,6}$, Liz Isenring ${ }^{7,8}$, Jenelle Loeliger ${ }^{9}$, \\ Belinda Steer ${ }^{9}$, Merran Findlay $^{10}$ \\ ${ }^{1}$ Institute for Physical Activity and Nutrition, Deakin University, Geelong, Australia \\ nicole.kiss@deakin.edu.au \\ ${ }^{2}$ Department of Cancer Experiences Research, Peter MacCallum Cancer Centre, Melbourne, Australia \\ ${ }^{3}$ School of Human Movement and Nutrition Sciences, University of Queensland, St Lucia, Australia \\ j.bauer1@uq.edu.au \\ ${ }^{4}$ Victorian Comprehensive Cancer Centre anna.boltong@unimelb.edu.au \\ ${ }^{5}$ Melbourne School of Population and Global Health, University of Melbourne, Melbourne, Australia \\ ${ }^{6}$ Nutrition and Dietetics Department, Royal Brisbane and Women's Hospital, Brisbane, Australia \\ Teresa.Brown@health.qld.gov.au \\ ${ }^{7}$ Faculty of Health Sciences and Medicine, Bond University, Robina, Australia lisenrin@bond.edu.au \\ ${ }^{8}$ Department of Nutrition and Dietetics, Princess Alexandra Hospital, Brisbane, Australia \\ ${ }^{9}$ Nutrition and Speech Pathology Department, Peter MacCallum Cancer Centre, Melbourne, Australia \\ jenelle.loeliger@petermac.org belinda.steer@petermac.org
}

${ }^{10}$ Royal Prince Alfred Hospital, Camperdown, Australia Merran.Findlay@health.nsw.gov.au

Corresponding author: Dr Nicole Kiss, Institute for Physical Activity and Nutrition, Deakin University, 221 Burwood Highway, Burwood, Victoria, Australia 3125. Email: nicole.kiss@deakin.edu.au, Phone: +61392468858.

Key words: cancer, oncology, malnutrition, sarcopenia, awareness, survey

\section{Acknowledgements}

The authors would like to thank the Clinical Oncology Society of Australia and Cancer Council Victoria for their distribution of the survey. We also acknowledge the clinicians who participated in the study. Dr Kiss is supported by a Victorian Cancer Agency Nursing and Allied Health Clinical Research Fellowship. 


\begin{abstract}
Purpose

Cancer-related malnutrition and sarcopenia have severe negative consequences including reduced survival and reduced ability to complete treatment. This study aimed to determine the awareness, perceptions and practices of Australian oncology clinicians regarding malnutrition and sarcopenia in people with cancer.
\end{abstract}

\title{
Methods
}

A national cross-sectional survey of Australian cancer clinicians was undertaken between November 2018 and January 2019. The 30-item online purpose-designed survey was circulated through professional organisations and health services.

\section{Results}

The 111 participants represented dietetic (38\%), nursing (34\%), medical (14\%) and other allied health (14\%) clinicians. Overall, $86 \%$ and $88 \%$ clinicians were aware of accepted definitions of malnutrition and sarcopenia, respectively. Perception of responsibility for identification of these conditions varied across participants, although 93\% agreed this was a component of their role. However, $21 \%$ and $43 \%$ of clinicians had limited or no confidence in their ability to identify malnutrition and sarcopenia, respectively. Common barriers to the identification and management of malnutrition were access to the tools or skills required and a lack of services to manage malnourished patients. Common barriers to identification of sarcopenia were lack of confidence and lack of services to manage sarcopenic patients. Enablers for identification and management of malnutrition and sarcopenia were variable, however training and protocols for management ranked highly.

\section{Conclusion}

While awareness of the importance of cancer-related malnutrition and sarcopenia are high, participants identified substantial barriers to delivering optimal nutrition care. Guidance at a national level is recommended to strengthen the approach to management of cancer-related malnutrition and sarcopenia. 


\section{Introduction}

Globally, the incidence of cancer is predicted to exceed 17 million people by 2020 [1], while currently in Australia, more than 144,000 new cases of cancer are diagnosed each year [2]. Cancer-related malnutrition affects approximately $30 \%$ to $40 \%$ of all people with cancer, however certain diagnoses including colorectal, lung, head and neck, upper gastrointestinal and haematological malignancies confer greater malnutrition risk $[3,4]$. Of the expected new cases in Australia annually, over $40 \%$ are cancer diagnoses associated with the highest risk of malnutrition [2]. Research over the past two decades has consistently demonstrated cancer-related malnutrition to be associated with reduced survival, poor function, reduced quality of life and higher health care costs [5-9]. Cancer-related sarcopenia is a condition characterised by loss of skeletal muscle mass and strength and is a key component of cancer-related malnutrition as reflected in the recent Global Leadership Initiative of Malnutrition (GLIM) criteria for diagnosis of malnutrition [10]. The adverse outcomes associated with cancer-related malnutrition are primarily due to depletion of skeletal muscle which may develop due to alterations in metabolism and utilisation of nutrients, inflammation, or as a consequence of treatment toxicities, in particular gastrointestinal side effects, impacting the ability to ingest sufficient nutrition [11]. While similarities exist, these conditions differ to cancer-related cachexia which is characterised by complex metabolic changes [12].

A multitude of evidence-based guidelines are available internationally to support the nutritional management of people with cancer [5-9]. These guidelines contain strong recommendations for the effectiveness of nutrition screening, assessment and treatment, if provided early in the disease course, to prevent and manage cancer-related malnutrition and sarcopenia supported largely by moderate levels of evidence [5-9]. Individualised combinations of dietary counselling, oral supplementation and enteral nutrition are shown to improve nutritional intake, minimise weight loss, improve quality of life and function and reduce unplanned hospital admissions [5-9]. However, integration of these practices into routine care remains highly variable [13].

It stands to reason that identification of malnutrition through recognised screening and assessment processes is likely to be important in preventing the severe consequences of cancer-related malnutrition and sarcopenia. A widespread appreciation of how to identify 
cancer-related malnutrition and sarcopenia across multidisciplinary oncology teams would contribute to appropriate and timely referrals for treatment. Despite the severe consequences, little is currently known about Australian oncology clinicians' awareness of these conditions and practices relating to their identification and management. This study aimed to determine awareness, perceptions and practices regarding cancer-related malnutrition and sarcopenia among Australian oncology health professionals.

\section{Methods}

Design and setting

A national cross-sectional survey of Australian cancer clinicians was undertaken between November 2018 and January 2019. Eligible participants included clinicians from an allied health, medical or nursing background who work with adults with cancer in an Australian health care setting and were over 18 years of age. The 30 -item online survey was circulated through the membership of professional cancer organisations (Clinical Oncology Society of Australia, Cancer Council Victoria Clinical Network), via the social media platform Twitter and to the professional networks of the research team via email invitation which included a link to the survey in the Qualtrics ${ }^{\mathrm{XM}}$ program (Qualtrics, Utah, USA). Ethical approval was obtained from the [REMOVED FOR BLIND REVIEW] Health Ethics Advisory Group (Ref: 163_208_181116) prior to data collection. Informed consent was obtained through a participant information sheet in Qualtrics prior to commencing the survey.

\section{Survey instrument}

A purpose designed survey was developed by the research team, who comprise clinical and research dietitians with a minimum of 15 years' expertise in nutrition and cancer, based on key evidence-based guidelines and literature $[5,8,9,14]$. The 30-item survey included questions on participant demographics and questions relating to four key areas: 1 ) awareness and understanding of malnutrition and sarcopenia, 2) perception of responsibility, 3) confidence in identification and appropriate management and 4) barriers and enablers to identification and appropriate management (supplementary file 1). All 
members of the research team trialled the survey questions to test face validity to ensure readability and clarity of content and structure.

Where participants were asked to choose statements that best represented their understanding of malnutrition or sarcopenia, potential responses included currently accepted definitions from professional organisations [10, 15-17]. Importance of malnutrition or sarcopenia in the context of cancer management were assessed on a 5-point Likert scale (1 = Extremely important, 2 = Very important, 3 = Moderately important, 4 = Somewhat important, 5 = Not at all important). Level of agreement with statements regarding perception of responsibility were assessed on a 5-point Likert scale ( 1 = Strongly agree, 2 = Agree, 3 = Neutral, 4 = Disagree, 5 = Strongly disagree). Confidence in identification of malnutrition and sarcopenia were assessed on a 4-point Likert scale (1 = Completely confident, 2 = Mostly confident, 3 = Equally confident/Not confident, $4=$ Not at all confident).

\section{Statistical analysis}

Data analysis was conducted using SPSS version 25 (IBM SPSS, Chicago, IL, USA). Descriptive statistics were used to summarise participant demographic characteristics and responses to survey questions. Cross tabulations were used to calculate relative frequency according to years of practice, discipline and tumour stream that comprised the health professional's main focus of practice. Tumour stream was recoded as high or low nutritional risk based on published prevalence of malnutrition [3, 4]. High risk included haematology, head and neck, lower gastrointestinal, lung, upper gastrointestinal and 'other' for which responses were palliative care or the health professional reported practising across all tumour streams. Low risk included breast, genitourinary, gynaecology, melanoma/ skin, neuro-oncology and sarcoma. Confidence was recoded into a binary variable (confident or not confident). Confident included responses indicating completely confident or mostly confident. Not confident included responses indicating equally confident/ not confident or not at all confident. Bivariate analysis was performed using Pearson's chi-squared tests. Fisher's exact test was used where recommended cell counts were not met for categorical variables. Alpha was set at 0.05 (two-tailed) for all analyses. 


\section{Results}

Participant characteristics

A total of 111 cancer clinicians from a range of disciplines participated in the survey (Table 1). The majority of participants were 40 years of age or over $(55 \%, n=61)$, worked in public hospital settings $(81 \%, n=90)$, in metropolitan areas $(76 \%, n=85)$, had over 10 years of experience in cancer care $(51 \%, n=56)$, and spent more than $75 \%$ of their time working in oncology $(67 \%, n=75)$. Practice areas were represented across all tumour types.

\section{INSERT TABLE 1 HERE}

\section{Awareness and understanding of malnutrition and sarcopenia}

Overall, 96 (86\%) participants identified a current accepted criterion for diagnosing malnutrition. Of the remaining 15 participants, two (2\%) selected a BMI $<18.5 \mathrm{~kg} / \mathrm{m}^{2}$ alone as their understanding of malnutrition, seven (6\%) participants were unsure what malnutrition was, and five (4\%) participants did not respond to this question. Similarly, 98 (88\%) participants identified a current accepted criterion for diagnosing sarcopenia. Of the remaining 13 participants, eight (7\%) participants were unsure what sarcopenia was, and five (4\%) participants did not respond to this question. The majority of participants $(N=99$, $89 \%)$ rated malnutrition and sarcopenia as extremely or very important in the overall management of people with cancer. Likewise, the majority of participants ( $N=9586 \%$ ) rated malnutrition and sarcopenia as extremely or very important in the supportive care management of people with cancer. No participants rated malnutrition and sarcopenia as not at all important in either the overall or supportive care management of people with cancer. Responses were not statistically different for correct identification of malnutrition or sarcopenia, or rating of their importance in overall or supportive care cancer management when compared by years of practice, tumour stream area of work (high versus low risk) or discipline. 
Perception of responsibility was high, with 93 (84\%) participants agreeing or strongly agreeing it was part of their role to recognise if a patient was malnourished or sarcopenic and initiate appropriate management. Only one participant strongly disagreed with this statement. The majority of participants believed the responsibility for identifying patients with malnutrition or sarcopenia lay with all health professionals (60\%), followed by the medical team (40\%) and dietitians (37\%) (Table 2). For treatment of malnutrition or sarcopenia the majority of participants believed responsibility lay with dietitians, followed by all health professionals and the medical team (Table 2). Overall, 100 (90\%) of participants reported discussing nutrition with their patients. In the majority of cases this occurred throughout the cancer journey.

\section{INSERT TABLE 2 HERE}

\section{Confidence in identification and management of malnutrition and sarcopenia}

Responses from all participants showed that $82(74 \%)$ participants were confident in identifying malnutrition with only $4(4 \%)$ not confident. However, when it came to identifying sarcopenia, 59 (53\%) of participants reported being confident and 16 (14\%) were not confident. In total, 73 (66\%) participants were aware of evidence-based guidelines for the nutritional management of people with cancer. The guidelines with the highest awareness among participants were local Australian guidelines produced by the Clinical Oncology Society of Australia (COSA) (49\%) and the Dietitians Association of Australia (DAA) (41\%), (supplementary file 2). Responses were not statistically different regarding confidence in identification of malnutrition or sarcopenia when compared by years of practice or tumour stream area of work (high vs low risk). However, responses were significantly different for confidence in both malnutrition and sarcopenia identification when compared by discipline. Dietitians were the discipline with the highest proportion of participants (98\%) confident in identifying malnutrition and physiotherapists were the discipline with the highest proportion of participants (80\%) confident in identifying sarcopenia (Supplementary file 3). 


\section{Barriers and enablers}

Regarding identification of malnutrition or sarcopenia, 43 (39\%) and $26(23 \%)$ of participants, respectively, reported no barriers in their health service. While, 19 (17\%) and $28(25 \%)$, respectively, were not aware of any enablers to malnutrition or sarcopenia identification at their health service. Barriers and enablers to identification of malnutrition and sarcopenia were ranked similarly with access to resources, lack of confidence and time being the greatest barriers, while protocols to support practice and training were the greatest enablers (Table 3). With respect to management of malnutrition or sarcopenia, 40 (36\%) and 29 (26\%) of participants, respectively, reported no barriers in their health service. While, $19(17 \%)$ and $30(27 \%)$ were not aware of any enablers to the management of malnutrition or sarcopenia, respectively, at their health services. Barriers to management of malnutrition and sarcopenia were also ranked similarly with lack of services to manage these conditions and knowledge and skills to provide appropriate care being the most frequently reported barriers. Enablers for the management of malnutrition and sarcopenia were more variable. Appropriate resources and implementation of protocols or pathways were ranked highly for both conditions, but training was the most frequently reported enabler for the management of sarcopenia. Overall, a low proportion of participants reported no barriers to identification ( $n=43,39 \%$ ) or management $(n=40,36 \%)$ of malnutrition at their health service. Likewise, there was a low proportion reporting no barriers to identification $(n=26,23 \%)$ or management $(n=29,26 \%)$ of sarcopenia.

Conversely, there was also a low proportion of participants reporting an absence of enablers to identification ( $n=19,17 \%)$ or management $(n=19,17 \%)$ of malnutrition at their health service. Similarly, a low proportion reported an absence of enablers to sarcopenia identification ( $n=28,25 \%)$ or management $(n=30,27 \%)$.

INSERT TABLE 3 HERE

\section{Discussion}


To our knowledge this study is the first to survey cancer clinicians from multiple disciplines and investigate awareness, perceptions and practices in regards to both cancer-related malnutrition and sarcopenia. The important role many disciplines play in the identification and management of cancer-related malnutrition and sarcopenia is reflected in several evidence-based guidelines on nutrition and cancer where the impact of nutrition screening, swallow function, symptom management and exercise are recognised $[5,6,8]$. The survey revealed high awareness and understanding of both conditions, with the vast majority of clinicians able to recognise accepted definitions of malnutrition and sarcopenia as well as their importance in the context of cancer care. Consistent with a multidisciplinary approach, there was a strong perception of responsibility across disciplines for recognising the presence of malnutrition or sarcopenia and initiating appropriate management.

Integral to timely management is clinician confidence in identifying cancer-related malnutrition and sarcopenia. The findings of this study revealed confidence in identifying malnutrition is understandably high among dietitians, with substantially lower confidence of $50 \%$ to $74 \%$ reported by other disciplines. This is in agreement with a previous study of medical oncologists in the United Kingdom [18], where an even greater proportion (80\%) lacked confidence in their ability to recognise malnutrition. Conversely, almost half the participants lacked confidence in identifying sarcopenia, suggesting less familiarity with sarcopenia among cancer clinicians. This is perhaps not surprising when we consider the literature relating to the adverse outcomes of cancer malnutrition dates back over 80 years [19], while the prognostic implications of sarcopenia have been a more recent focus in the past 10 years $[20,21]$. There are a number of validated nutrition screening tools to identify people at risk of malnutrition, including the malnutrition screening tool (MST), Malnutrition Universal Screening Tool (MUST), in cancer populations $[8,9]$. While screening tools for sarcopenia, such as the SARC-F, are beginning to be studied in people with cancer, they are yet to be validated in cancer populations [22-24]. When a risk of malnutrition is identified a comprehensive nutrition assessment including evaluation of body composition and muscle mass is completed, aligning with current evidence-based guideline recommendations and the GLIM criteria and facilitating the diagnosis of both malnutrition and sarcopenia $[6,10$, 16]. Professional development resources with a particular focus on the identification and 
management of sarcopenia in people with cancer, and its relationship to malnutrition and cachexia may be required to support improved clinician confidence.

A number of barriers and enablers to identification and management of malnutrition were reported with the most common including lack of knowledge, skills, confidence and time. Our findings in a multidisciplinary sample reflect the findings of previous studies and systematic reviews investigating nutrition screening and support practices in single discipline studies of medical oncologists, nurses working in acute health care and dietitians working with community living older adults $[18,25,26]$. Participants in these previous studies consistently report that lack of guidelines (70\%), time (43-55\%) and knowledge (17$60 \%)$ regarding malnutrition are the most common barriers, and organisation support through training programs and nutrition policies (51\%) are the most common enablers to optimal nutrition care. Lack of evidence was also identified as a barrier for identifying and managing sarcopenia, likely reflecting the more recent nature of the literature in this area related to cancer. Similarly, the most common enablers of training, protocols or pathways, role delineation and adequate resourcing also reflect previous literature and were similar for malnutrition and sarcopenia $[18,25,26]$. Of note, previous studies have not examined barriers and enablers to identification and management of sarcopenia. The findings of this study suggest barriers and enablers are similar across both conditions.

Strong evidence exists to support the importance of early recognition and treatment of cancer-related malnutrition and sarcopenia, nevertheless achieving consistent practice across professions and health services is challenging. The delay in the translation of research knowledge into clinical practice is well known, with specific strategies and support required for implementation [27]. Clinicians and health services require assistance and resources to achieve optimal practices in identifying and treating cancer-related malnutrition and sarcopenia. Flexibility in approaches may be required, such as patient-led nutrition screening using tools such as the MST which has been shown to be a reliable method with high patient acceptability [28]. At the local health service level, protocols and pathways implemented in specific cancer populations have demonstrated improved adherence to evidence-based nutrition care $[29,30]$. A number of studies investigating enablers to optimal nutrition care in cancer report that clinicians seek protocols and pathways at a national level to guide practice [31,32]. Such protocols or pathways would ideally provide 
an overarching framework for nutrition care with capacity for adaptability to specific health service cultures and structures with examples available in haematology, head and neck, and general oncology populations [29, 30, 33].

Internationally, a number of organisations have invested time and resources into improving recognition and care of malnutrition related to chronic disease or more specifically in cancer. These include the British Association of Parenteral and Enteral Nutrition (BAPEN), the European Society of Clinical Nutrition and Metabolism (ESPEN), the Dutch Malnutrition Steering Group, Macmillan Cancer Support, and in Australia, the Victorian Cancer Malnutrition Collaborative (VCMC), Clinical Oncology Society of Australia (COSA), Dietitians Association of Australia (DAA) and the AIOM-SINPE-FAVO working group [7-9, 13, 34-37]. Commonalities in approaches across these groups involve multidisciplinary engagement, government advocacy, and clinician and patient resources. However, the results of our study suggest further efforts are still required to overcome the barriers that persist in order to optimise nutritional care and leverage the enablers clinicians have identified. The findings of this study will help inform the development of a national multidisciplinary strategic approach to optimising cancer nutrition care in Australia. While this study did not involve people with cancer, further initiatives to improve nutritional care should consider their involvement as an important component to driving changes in practice.

It is acknowledged this study has some limitations. Firstly, the recruitment strategy did not support calculation of a response rate and we are therefore unable to determine if our sample is representative of multidisciplinary oncology clinicians across Australia. Secondly, the sample size is relatively small and is underrepresented by rural clinicians and allied health professionals other than dietitians. Although the discipline proportions are similar to those reported in previous surveys of multidisciplinary oncology clinicians [38]. Strengths of the study are the comprehensive assessment of awareness, perceptions and practices across multiple disciplines and the first to develop this understanding for both cancerrelated malnutrition and sarcopenia.

\section{Conclusions}

This study demonstrated high awareness and recognition of the importance of cancerrelated malnutrition and sarcopenia in the context of cancer care. Overall, oncology 
clinicians acknowledged their individual responsibility in the identification and management of these conditions. The majority of participants reported barriers still existed to identification and management, however a high proportion also acknowledged the presence of enablers within their organisations. A strategic and multidisciplinary approach to the development of resources and guidance at a national level is recommended to strengthen and streamline the approach to management of cancer related malnutrition and sarcopenia.

\section{Conflict of interest}

None to declare. The authors have full control of the data and agree to allow the journal to review the data if requested.

\section{References}

1. International Agency for Research on Cancer Globocan Database. Estimated Incidence, Mortality and Prevalence Worldwide in 2012 Fact Sheet. 2012; http://globocan.iarc.fr/Pages/fact sheets cancer.aspx. Accessed 3 August 2019.

2. Australian Institute of Health and Welfare, Cancer in Australia 2019. 2019, Australian Institute of Health and Welfare: Canberra.

3. Hébuterne X, Lemarié E, Michallet M, de Montreuil CB, Schneider SM, Goldwasser F (2014). Prevalence of Malnutrition and Current Use of Nutrition Support in Patients With Cancer. JPEN 38(2):196-204.

4. Marshall KM, Loeliger J, Nolte L, Kelaart A, Kiss NK (2019). Prevalence of malnutrition and impact on clinical outcomes in cancer services: A comparison of two time points. Clin Nutr 38(2):644 - 51.

5. Academy of Nutrition and Dietetics. Oncology Evidence-Based Nutrition Practice Guideline. http://andevidenceanalysislibrary.com. Accessed 6 Auguets 2019.

6. Arends J, Bachmann P, Baracos V, Barthelemy N, Bertz H, Bozzetti F, et al (2017). ESPEN guidelines on nutrition in cancer patients. Clin Nutr 36(1):11-48.

7. Cotogni, P, Pedrazzoli P, De Waele E, Aprile E, Farina G, Stragliotto S, De Lorenzo F, Caccialanza R (2019). Nutritional Therapy in Cancer Patients Receiving Chemoradiotherapy: Should We Need Stronger Recommendations to Act for Improving Outcomes? J Cancer 10(18): 4318-4325.

8. Findlay M, Bauer J, Brown T, Davidson W, Hill J, Isenring E, et al. Evidence based practice guidelines for the nutritional managment of adult patients with head and neck cancer.

http://wiki.cancer.org.au/australia/COSA:Head and neck cancer nutrition guidelines. Accessed 6 August 2019 
9. Isenring E, Zabel R, Bannister M, Brown T, Findlay M, Kiss N, et al (2013). Updated evidence-based practice guidelines for the nutritional management of patients receiving radiation therapy and/or chemotherapy. Nutr Diet 70(4):312 - 24.

10. Cederholm T, Jensen GL, Correia MITD, Gonzalez MC, Fukushima R, Higashiguchi T, et al (2019). GLIM criteria for the diagnosis of malnutrition: A consensus report from the global clinical nutrition community. Clin Nutr 38(1):1-9.

11. Baracos VE (2018). Cancer-associated malnutrition. Eur J Clin Nutr 72(9):1255-9.

12. Fearon K, Strasser F, Anker SD, Bosaeus I, Bruera E, Fainsinger RL, et al (2011). Definition and classification of cancer cachexia: an international consensus. Lancet Oncol 12(5):489-95.

13. Arends J, Baracos V, Bertz H, Bozzetti F, Calder PC, Deutz NEP, et al (2017). ESPEN expert group recommendations for action against cancer-related malnutrition. Clin Nutr 36(5):1187-96.

14. Arends J, Bodoky G, Bozzetti F, Fearon K, Muscaritoli M, Selga G, et al (2006). ESPEN guidelines on enteral nutrition: non-surgical oncology. Clin Nutr 25:249 - 59.

15. Baracos VE, Mazurak VC, Bhullar AS (2018). Cancer cachexia is defined by an ongoing loss of skeletal muscle mass. Ann Pall Med 8(1):3-12.

16. Cederholm T, Bosaeus I, Barazzoni R, Bauer J, Van Gossum A, Klek S, et al (2015). Diagnostic criteria for malnutrition - An ESPEN Consensus Statement. Clin Nutr 34(3):335-40.

17. Cruz-Jentoft AJ, Bahat G, Bauer J, Boirie Y, Bruyère O, Cederholm T, et al (2019). Sarcopenia: revised European consensus on definition and diagnosis. Age and ageing 48(1):16-31.

18. Spiro A, Baldwin C, Patterson A, Thomas J, Andreyev HJN (2006). The views and practice of oncologists towards nutritional support in patients receiving chemotherapy. Br J Cancer 95(4):431-4.

19. Warren S (1932). The immediate cause of death in cancer. Am J Med Sciences 184:610 - 3.

20. Blauwhoff-Buskermolen S, Versteeg KS, Schueren MAEdvd, Braver NRd, Berkhof J, Langius JAE, et al (2016). Loss of Muscle Mass During Chemotherapy Is Predictive for Poor Survival of Patients With Metastatic Colorectal Cancer. J Clin Oncol 34(12):1339-44.

21. Prado CMM, Lieffers JR, McCargar LJ, Reiman T, Sawyer MB, Martin L, et al (2008). Prevalence and clinical implications of sarcopenic obesity in patients with solid tumours of the respiratory and gastrointestinal tracts: a population-based study. Lancet Oncol 9(7):629-35.

22. Malmstrom TK, Morley JE (2013). SARC-F: A Simple Questionnaire to Rapidly Diagnose Sarcopenia. J Am Medical Directors Association 14(8):531-2.

23. Noronha Barrére A, Lopes G, de Assis T, Fraga Piovacari S.M, Toledo D.O. (2018). Identification of Sarcopenia Risk in Oncology Outpatients using the SARC-F Method. J Nutr Health \& Food Science $6(5): 1-5$.

24. Wang B, Thapa S, Zhou T, Lui H, Li L, Peng G, Yu S (2019). Cancer-related fatigue and biochemical parameters among cancer patients with different stages of sarcopenia. Support Care Cancer doi: 10.1007/s00520-019-04717-0. 
25. Craven DL, Pelly FE, Isenring E, Lovell GP (2017). Barriers and enablers to malnutrition screening of community-living older adults: a content analysis of survey data by Australian dietitians. Austr J Primary Health 23(2):196-201.

26. Green SM, James EP (2013). Barriers and facilitators to undertaking nutritional screening of patients: a systematic review. J Hum Nutr Diet 26(3):211-21.

27. Morris Z.S, Wooding S, Grant J (2011). The answer is 17 years, what is the question: understanding time lags in translational research. J Royal Soc Medicine 104(12):510-520.

28. Di Bella A, Blake C, Young A, Pelecanos A, Brown T (2018). Reliability of Patient-Led Screening with the Malnutrition Screening Tool: Agreement between Patient and Health Care Professional Scores in the Cancer Care Ambulatory Setting. J Academy Nutr Diet 118(6):1065-71.

29. Atkins L, Steer B, Ray H, Kiss N (2019). Implementing and sustaining an evidence-based nutrition service in a haematology unit for autologous stem cell transplant patients. Support Care Cancer 27(3):951-8.

30. Kiss N, Krishnasamy M, Loeliger J, Granados A, Dutu G, Corry J (2012). A dietitian-led clinic for patients receiving (chemo)radiotherapy for head and neck cancer. Support Care Cancer 20:2111 - 20.

31. Caccialanza R, Cereda E, Pinto C, Cotogni P, Farina G, Gavazzi C, et al (2016). Awareness and consideration of malnutrition among oncologists: Insights from an exploratory survey. Nutrition 32(9):1028-32.

32. Martin L, de van der Schueren MAE, Blauwhoff-Buskermolen S, Baracos V, Gramlich L (2016). Identifying the Barriers and Enablers to Nutrition Care in Head and Neck and Esophageal Cancers. JPEN 40(3):355-66.

33. Victorian Cancer Malnutrition Collaborative. The CanEAT pathway. Available from: https://www.petermac.org/CanEATpathway. Accessed 30 th January 2020.

34. British Association of Parenteral and Enteral Nutrition (2018). Introduction to Malnutrition 2018, https://www.bapen.org.uk/malnutrition-undernutrition/introduction-to-malnutrition. Accessed 8 August 2019.

35. Dutch Malnutrition Steering Group (2019). Fight Malnutrition https://www.fightmalnutrition.eu/. Accessed 8 Auguest 2019.

36. Macmillan Cancer Support (2019). Principles and guidance for prehabilitation within the management and support of people with cancer.

37. Victorian Cancer Malnutrition Collaborative. Investigating Practices Relating to Malnutrition in Victorian Cancer Services: Victorian Department of Health and Human Services https://www2.health.vic.gov.au/about/health-strategies/cancer-care/cancer-projects/investigatingpractices-relating-to-malnutrition-in-victorian-cancer-services. Accessed 8 August 2019.

38. Chan RJ, Yates P, Li Q, Komatsu H, Lopez V, Thandar M, et al (2017). Oncology practitioners' perspectives and practice patterns of post-treatment cancer survivorship care in the Asia-Pacific region: results from the STEP study. BMC Cancer 17(1):715-. 
This is a post-peer-review, pre-copyedit version of an article published in Supportive Care in Cancer. The final authenticated version is available online at: https://doi.org/10.1007/s00520-020-05371-7 